\title{
Baicalin serves a protective role in diabetic nephropathy through preventing high glucose-induced podocyte apoptosis
}

\author{
JINDONG LI ${ }^{1}$, YA LING ${ }^{2}$, SHENGNAN YIN ${ }^{3}$, SHUFANG YANG ${ }^{1}$, MIN KONG $^{1}$ and ZHIQIN LI ${ }^{1}$ \\ ${ }^{1}$ Department of Pharmacy, Hospital Affiliated 5 to Nantong University, Taizhou People's Hospital, Taizhou, Jiangsu 225300; \\ ${ }^{2}$ Department of Pharmacy, The First Affiliated Hospital of Soochow University, Suzhou, Jiangsu 215006; \\ ${ }^{3}$ Department of Pharmacy, Hospital of Traditional Chinese Medicine of Taizhou, Taizhou, Jiangsu 225300, P.R. China
}

Received September 3, 2019; Accepted March 24, 2020

DOI: $10.3892 /$ etm.2020.8701

\begin{abstract}
Diabetic nephropathy (DN) is one of the late complications of diabetes, which seriously affects the lives of patients. Baicalin (BA) is a flavone glycoside that has been identified to improve renal function in patients with DN. The present study aimed to investigate the roles and mechanisms of BA in DN. For that purpose, podocytes were cultured for $48 \mathrm{~h}$ under conditions of high glucose (HG; $30 \mathrm{mM}$ D-glucose) or normal glucose (NG; $5 \mathrm{mM}$ D-glucose). Then, the cells were treated with different concentrations of BA $(6.25,12.5$ and $25 \mu \mathrm{M})$ for $24 \mathrm{~h}$. Cell viability and apoptosis were determined using an MTT assay and flow cytometry, respectively. Protein and mRNA expression levels were analyzed using western blotting and reverse transcription-quantitative PCR, respectively. BA treatment was identified to promote the viability of podocytes and suppress cell apoptosis in a dose-dependent manner. Compared with the results in the $\mathrm{NG}$ group, $\mathrm{HG}$ stimulation significantly decreased the viability of podocytes and increased the apoptotic rate, whereas BA treatment following $\mathrm{HG}$ stimulation increased the viability of podocytes and decreased the apoptotic rate. Moreover, the effect of BA was revealed to be associated with the sirtuin $1 / \mathrm{NF}-\kappa \mathrm{B}$ signaling pathway in DN. In conclusion, the results of the present study suggested that BA treatment may significantly decrease HG-induced podocyte apoptosis, which indicated that BA might be a promising agent for DN treatment.
\end{abstract}

\section{Introduction}

Diabetic nephropathy (DN) is a serious and harmful chronic complication caused by diabetes and it is a common cause of

Correspondence to: Dr Zhiqin Li, Department of Pharmacy, Hospital Affiliated 5 to Nantong University, Taizhou People's Hospital, 399 Hailing South Road, Taizhou, Jiangsu 225300, P.R. China

E-mail: lizhiqin190902@163.com

Key words: diabetic nephropathy, baicalin, podocytes, sirtuin $1 / \mathrm{NF}-\kappa \mathrm{B}$ signaling pathway end-stage renal disease (1). Diabetes can cause glomerular damage, accompanied by proteinuria and subsequent tubulointerstitial lesions, which ultimately culminates in end-stage renal disease (2-4). At present, DN occurs in $20-40 \%$ of patients with diabetes (5). The development of DN takes years and involves numerous characteristic pathological changes, such as the excessive accumulation of the extracellular matrix, glomerular sclerosis, tubular dilatation and atrophy, and interstitial fibrosis (6). A major pathological feature of DN is the abnormal apoptosis of podocytes. In a previous study, it was identified that the apoptosis of podocytes was associated with the decreased expression levels of multiple proteins, including podocin, nephrin and Slit-associated proteins (7), which resulted in considerable proteinuria in $\mathrm{DN}$ (8). Therefore, it is important to prevent podocyte apoptosis and cure $\mathrm{DN}$ at an early stage.

Baicalin (BA; 5,6,7-trihydroxyflavone), a herbal medicine found in the Chinese Pharmacopoeia, is one of the main flavonoids purified from the roots of Astragalus membranaceus (9). It has been reported that BA exhibits significant anticancer effects in a variety of malignant tumors, including colorectal (10), breast (11) and lung cancer (12), where it served an important role in regulating cell growth. In addition, in a previous study, it was identified that BA treatment improved the renal function of patients with DN and delayed the progression of DN through both anti-inflammatory and antioxidation mechanisms (13). However, to the best of our knowledge, there are currently no existing studies on the effect of BA on high glucose (HG)-induced podocyte apoptosis.

Sirtuin 1 (SIRT1) is an important nicotinamide adenine dinucleotide-dependent nuclear histone deacetylase that has been identified to serve a crucial role in the vascular system through regulating cell proliferation and the cell cycle (14). It was previously demonstrated that SIRT1 served an important role in the pathogenesis of kidney disease (15). In fact, the activation of SIRT1 has been suggested to be a novel target for the treatment of patients with chronic kidney disease, including DN (16).

$\mathrm{NF}-\kappa \mathrm{B}$, one of the most important transcription factors in macrophages, extensively regulates cell proliferation, apoptosis and inflammatory cytokine expression (17), and it is also identified to be activated in diabetic conditions (18). 
The activated and phosphorylated form of NF- $\kappa \mathrm{B}$ subunits (p65 and p50) translocate to the nucleus, which indicates the activation of the NF- $\kappa B$ signaling pathway (19). Numerous reports have revealed that hyperglycemia-induced $\mathrm{DN}$ and the associated inflammation is induced by NF- $\mathrm{kB}$; the majority of proinflammatory cytokines are regulated by $\mathrm{NF}-\mathrm{\kappa B}$ transcription (20-22). Notably, NF- $\mathrm{BB}$ was identified to be involved in regulating apoptosis in podocytes $(21,22)$.

The present study aimed to investigate the effects of BA treatment on podocytes in vitro and to determine whether BA protected against DN through inhibiting HG-induced podocyte apoptosis. Furthermore, the underlying molecular mechanisms were analyzed to reveal the potential role and value of BA in DN. The results of the present study provided a theoretical basis, as well as treatment strategies for the treatment of DN.

\section{Materials and methods}

Cell culture and HG-induced podocyte injury model. The conditionally immortalized mouse podocyte MPC-5 cell line (cat no. C1389) were obtained from Shanghai Guandao Biological Engineering Co., Ltd. Cells were cultured in RPMI-1640 medium (Gibco; Thermo Fisher Scientific, Inc.), supplemented with 10\% FBS (Invitrogen; Thermo Fisher Scientific, Inc.) and $1 \%$ (v/v) penicillin-streptomycin (Gibco; Thermo Fisher Scientific, Inc.), and maintained in a humidified atmosphere containing $5 \% \mathrm{CO}_{2}$ at $37^{\circ} \mathrm{C}$.

For HG-induction, following $12 \mathrm{~h}$ of serum starvation, podocytes were cultured at $37^{\circ} \mathrm{C}$ for $48 \mathrm{~h}$ under conditions of HG (30 mM D-glucose; Sigma-Aldrich; Merck KGaA) or normal glucose (NG; 5 mM D-glucose; Sigma-Aldrich; Merck $\mathrm{KGaA})(23)$.

MTT assay. Cell viability was analyzed using an MTT assay (Beijing Solarbio Science \& Technology Co., Ltd.) according to the manufacturer's protocol. For MTT detection, after $12 \mathrm{~h}$ of serum starvation, podocytes were cultured for $48 \mathrm{~h}$ under conditions of HG (30 mM D-glucose) or NG (5 mM D-glucose). Podocytes $\left(5 \times 10^{4}\right.$ per well) were seeded into 96-well plates and treated with $6.25,12.5$ or $25 \mu \mathrm{M}$ BA (Sigma-Aldrich; Merck KGaA) at $37^{\circ} \mathrm{C}$ for $24 \mathrm{~h}$. Following the treatment, $20 \mu 1$ MTT reagent was added into each well and incubated for $4 \mathrm{~h}$ at $37^{\circ} \mathrm{C}$. Subsequently, $150 \mu 1 \mathrm{DMSO}$ was added into each well and shaken at $37^{\circ} \mathrm{C}$ for $15 \mathrm{~min}$. Finally, the optical density values were measured at $490 \mathrm{~nm}$ using a microplate reader.

Flow cytometric analysis of apoptosis. Following the treatment of podocytes with $6.25,12.5$ or $25 \mu \mathrm{M} \mathrm{BA}$, apoptosis was analyzed using flow cytometry. Briefly, the podocytes were digested from 6-well plates using trypsin, resuspended in fresh medium and centrifuged at $500 \mathrm{xg}$ for $5 \mathrm{~min}$ at room temperature. The supernatant was discarded and the pellet was resuspended in $1 \mathrm{X}$ binding buffer. The cells were subsequently stained with Annexin V-FITC solution and $\mathrm{PI}$ at $4^{\circ} \mathrm{C}$ for $15 \mathrm{~min}$ using the Annexin V-FITC Apoptosis Detection kit (Sigma-Aldrich; Merck KGaA) according to the manufacturer's protocol. Apoptotic cells were analyzed using a flow cytometer and the flow cytometry data were analyzed by FlowJo software (version 7.6.1; FlowJo LLC) to determine the percentage of apoptotic cells.

Western blotting. Following the treatment of podocytes with $6.25,12.5$ or $25 \mu \mathrm{M}$ BA, total protein was extracted from the conditionally immortalized mouse podocytes using RIPA lysis buffer (Beyotime Institute of Biotechnology). Total protein was quantified using a bicinchoninic acid assay kit (Beyotime Institute of Biotechnology) and $30 \mu \mathrm{g}$ protein/lane was separated by $10 \%$ SDS-PAGE. The separated proteins were subsequently transferred onto a PVDF membrane (EMD Millipore) and blocked at room temperature with $5 \%$ fat-free powdered milk dissolved in tris-buffered saline (TBS) containing $0.1 \%$ Tween for $1.5 \mathrm{~h}$. The membrane was incubated with the following primary antibodies at $4^{\circ} \mathrm{C}$ overnight: Anti-SIRT1 (1:1,000; Cell Signaling Technology, Inc.), anti-cleaved caspase-3 (1:1,000; Santa Cruz Biotechnology, Inc.), anti-pro-caspase-3 (1:1,000; Santa Cruz Biotechnology, Inc.), anti-GAPDH (1:5,000; Cell Signaling Technology, Inc.), anti-p65 (1:1,000; Abcam) and anti-phosphorylated (p)-p65 $(1: 1,000 ;$ Abcam). Following the primary antibody incubation, the membranes were incubated with a horseradish peroxidase-conjugated secondary antibody (cat no. ab7090; 1:2,000; Abcam) at room temperature for $2 \mathrm{~h}$. The protein bands were visualized using an enhanced chemiluminescence reagent (EMD Millipore) and GAPDH served as a loading control for normalization.

Reverse transcription-quantitative PCR (RT-qPCR). Following the treatment of podocytes with $6.25,12.5$ or $25 \mu \mathrm{M}$ BA, total RNA was extracted from podocytes using TRIzol ${ }^{\circledR}$ reagent on ice (Invitrogen; Thermo Fisher Scientific, Inc.), according to the manufacturer's protocol. Following the RNA extraction, the concentration of each sample was measured using an ultraviolet spectrophotometer. Total RNA was reverse transcribed into cDNA using a HiScipt II 1st Strand cDNA Synthesis Kit (Vazyme Biotech Co., Ltd.), according to the manufacturer's protocol. The following RT temperature protocol was used: $70^{\circ} \mathrm{C}$ for $5 \mathrm{~min}, 37^{\circ} \mathrm{C}$ for $5 \mathrm{~min}$ and $42^{\circ} \mathrm{C}$ for 60 min. qPCR was subsequently performed using a HiScript II One Step qRT-PCR SYBR Green kit (Vazyme Biotech Co., Ltd.) according to the manufacturer's protocol. The following thermocycling conditions were used for the qPCR: $95^{\circ} \mathrm{C}$ for $3 \mathrm{~min}$; followed by 40 cycles of $95^{\circ} \mathrm{C}$ for $30 \mathrm{sec}, 56^{\circ} \mathrm{C}$ for $30 \mathrm{sec}$ and $72^{\circ} \mathrm{C}$ for $30 \mathrm{sec}$. GAPDH served as the internal loading control for normalization. Primer sequences for PCR were listed as following: SIRT1 forward, 5'-AATCCAGTCATT AAAGGTCTACAA-3' and reverse, 5'-TAGGACCATTAC TGCCAGAGG-3'; p65 forward, 5'-CATGCGCTTCCGCTA CAAG-3' and reverse, 5'-GGTCCCGCTTCTTTACACAC-3' and GAPDH forward, 5'-CCATGGGGAAGGTGAAGGTC-3' and reverse, 5'-GAAGGGGTCATTGATGGCAAC-3'. Data were quantified using the $2^{-\Delta \Delta C q}$ method (24).

Statistical analysis. Experiments were repeated three times. Statistical analysis was performed using SPSS 22.0 software (IBM Corp.) and data are presented as the mean \pm SD. Statistical differences between groups were determined using one-way ANOVA with a Bonferroni post hoc test. $\mathrm{P}<0.05$ was considered to indicate a statistically significant difference. 


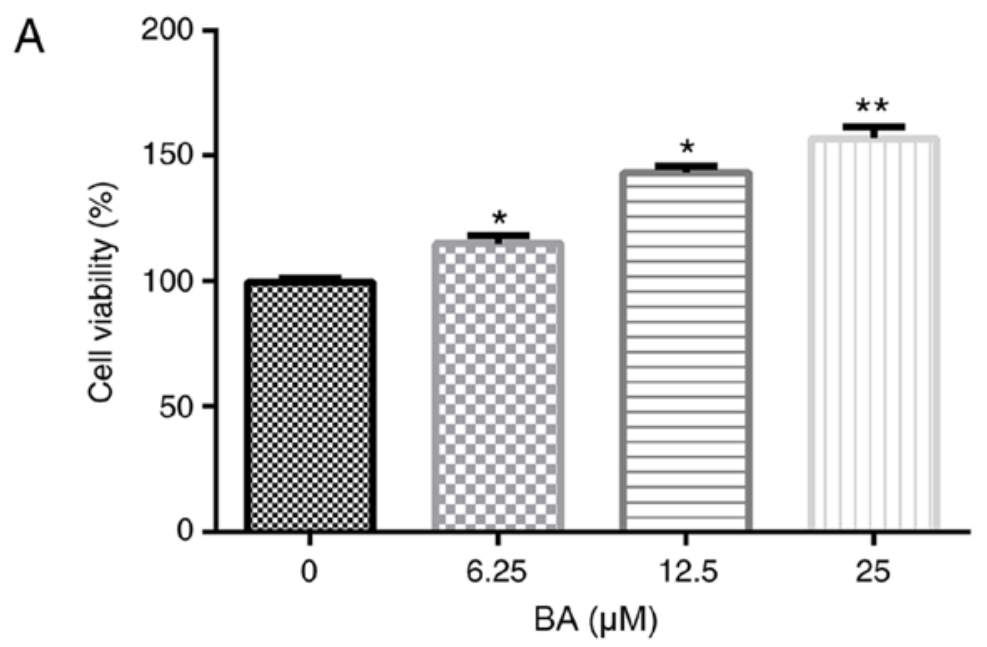

B

$\mathrm{BA}(\mu \mathrm{M})$
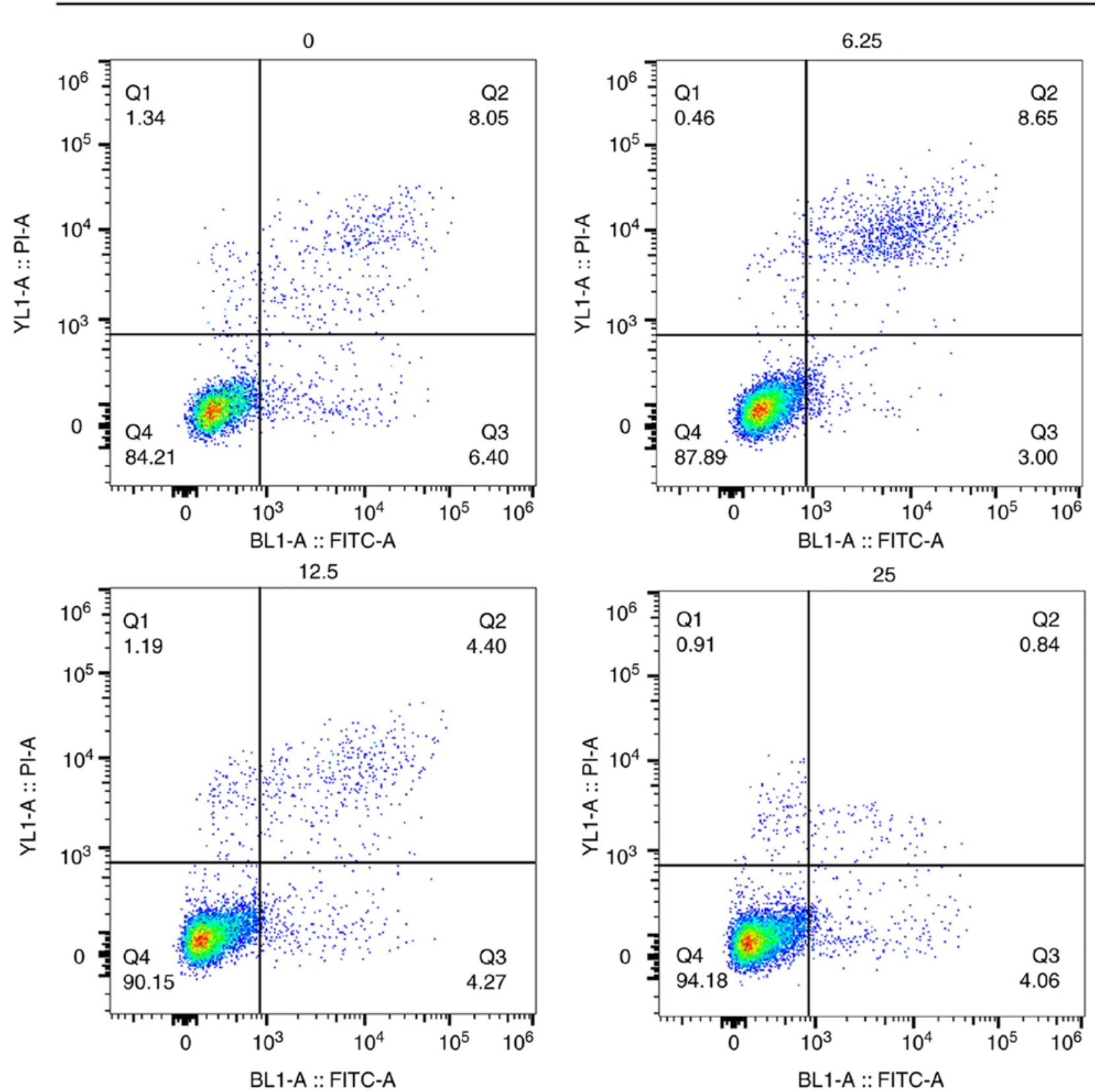

Figure 1. BA promotes podocyte viability and decreases apoptosis. Podocytes were treated with $6.25,12.5$ or $25 \mu \mathrm{M}$ BA for $24 \mathrm{~h}$. (A) MTT assay was used to determine the cell viability. (B) Apoptotic rate of podocytes was analyzed using flow cytometry. 


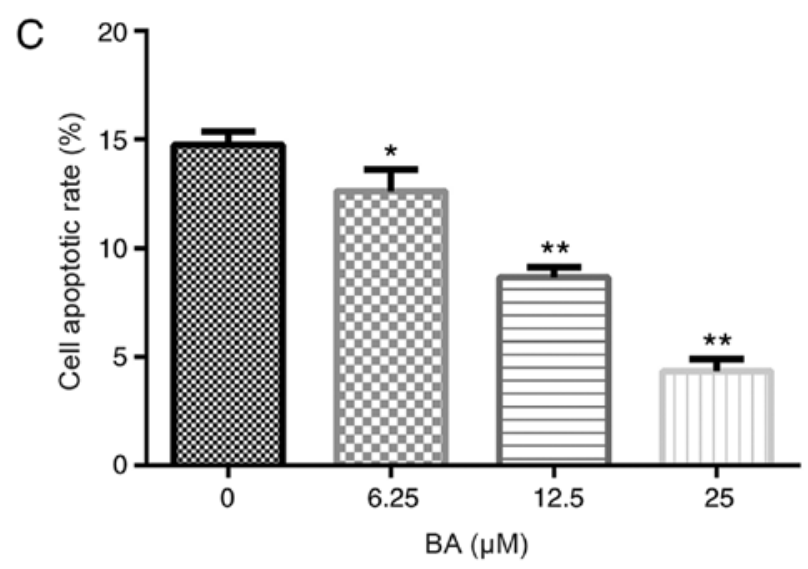

$\mathrm{D}$

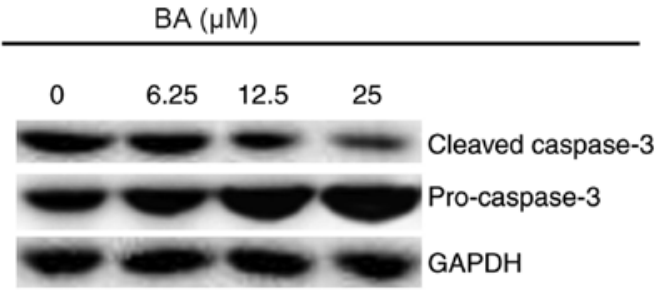

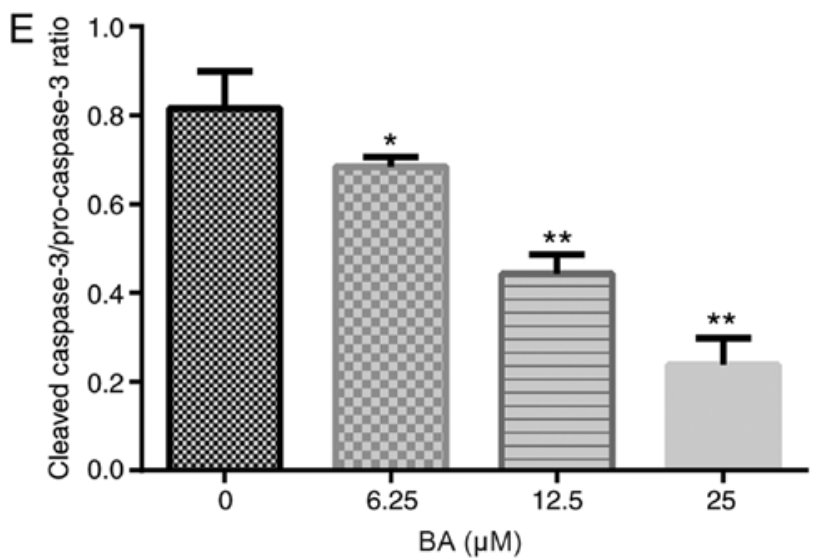

Figure 1. Continued. (C) Cell apoptotic rate was calculated. (D) Western blotting was used to analyze the protein expression levels of pro-caspase-3 and cleaved caspase-3. (E) Semi-quantification of the ratio between cleaved caspase-3/pro-caspase-3. All data are presented as the mean $\pm \mathrm{SD}$. ${ }^{*} \mathrm{P}<0.05$ and ${ }^{* * *} \mathrm{P}<0.01$ vs. $0 \mu \mathrm{M}$ BA treatment group. BA, baicalin; PI, propidium iodide.

\section{Results}

Effects of BA on podocyte viability and apoptosis. To investigate the effects of BA, an MTT assay and flow cytometry were performed to determine the cell viability and rate of apoptosis, respectively. Compared with the untreated group, BA significantly increased the viability of podocytes in a dose-dependent manner (Fig. 1A). In addition, compared with the untreated group, BA significantly inhibited cell apoptosis in a dose-dependent manner (Fig. 1B and C). The results from the western blotting also revealed that BA treatment reduced the cleaved-caspase-3/pro-caspase-3 ratio compared with that in the untreated cells (Fig. 1D and E). Taken together, these results suggested that BA treatment may increase cell viability and decrease the rate of apoptosis.

Effects of BA on the HG-induced podocyte injury model. To investigate the effects of BA on the HG-induced podocyte injury model, podocytes were cultured for $48 \mathrm{~h}$ under conditions of $\mathrm{HG}$ (30 mM D-glucose) or NG (5 mM D-glucose) following serum starvation for $12 \mathrm{~h}$. Then, the cells were treated with different concentrations of BA for $24 \mathrm{~h}$. The MTT assay demonstrated that HG stimulation significantly decreased the viability of podocyte cells, whereas the treatment of HG-induced podocytes with BA significantly weakened the effects of HG in a dose-dependent manner; a significantly

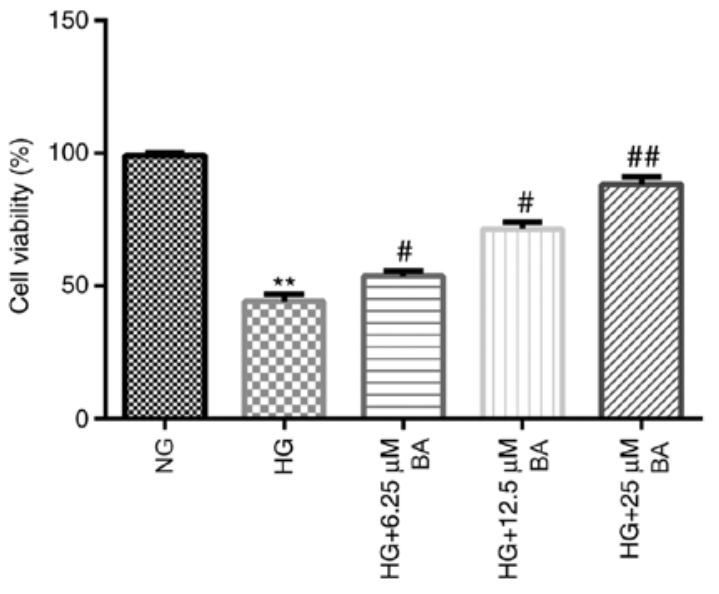

Figure 2. BA improves HG-induced decreased podocyte viability. Following serum starvation for $12 \mathrm{~h}$, podocytes were cultured with $\mathrm{HG}(30 \mathrm{mM}$ D-glucose) or normal glucose (5 mM D-glucose) for $48 \mathrm{~h}$ and then treated with $6.25,12.5$ or $25 \mu \mathrm{M}$ BA for $24 \mathrm{~h}$. An MTT assay was used to determine the cell viability. All data are presented as the mean $\pm \mathrm{SD} .{ }^{* *} \mathrm{P}<0.01$ vs. NG group. ${ }^{\#} \mathrm{P}<0.05$ and ${ }^{\# \#} \mathrm{P}<0.01$ vs. HG treatment group. BA, baicalin; HG, high glucose; NG, normal glucose.

increased cell viability was observed in the $\mathrm{HG}+\mathrm{BA}$-treated groups compared with the HG group (Fig. 2).

Flow cytometry and western blotting were subsequently performed to determine the rate of apoptosis and 
A

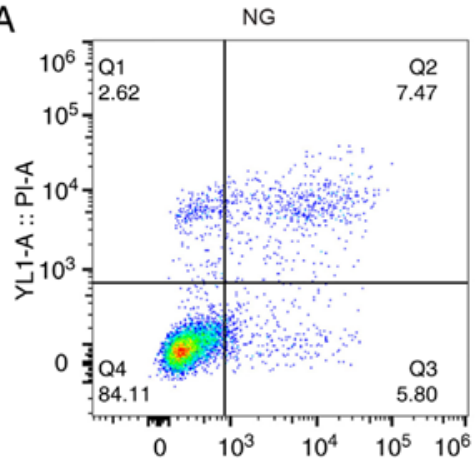

BL1-A :: FITC-A

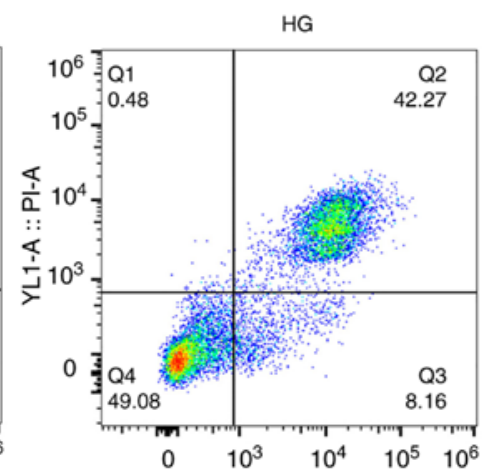

BL1-A : FITC-A

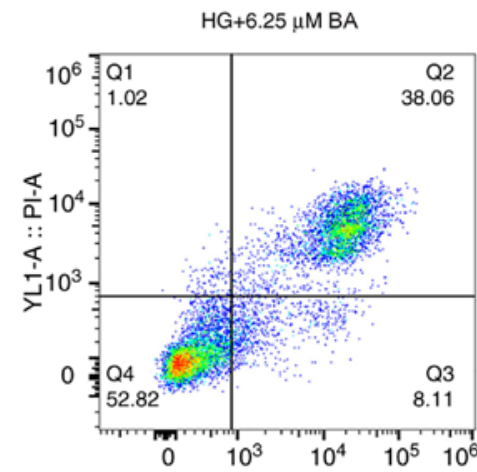

BL1-A :: FITC-A
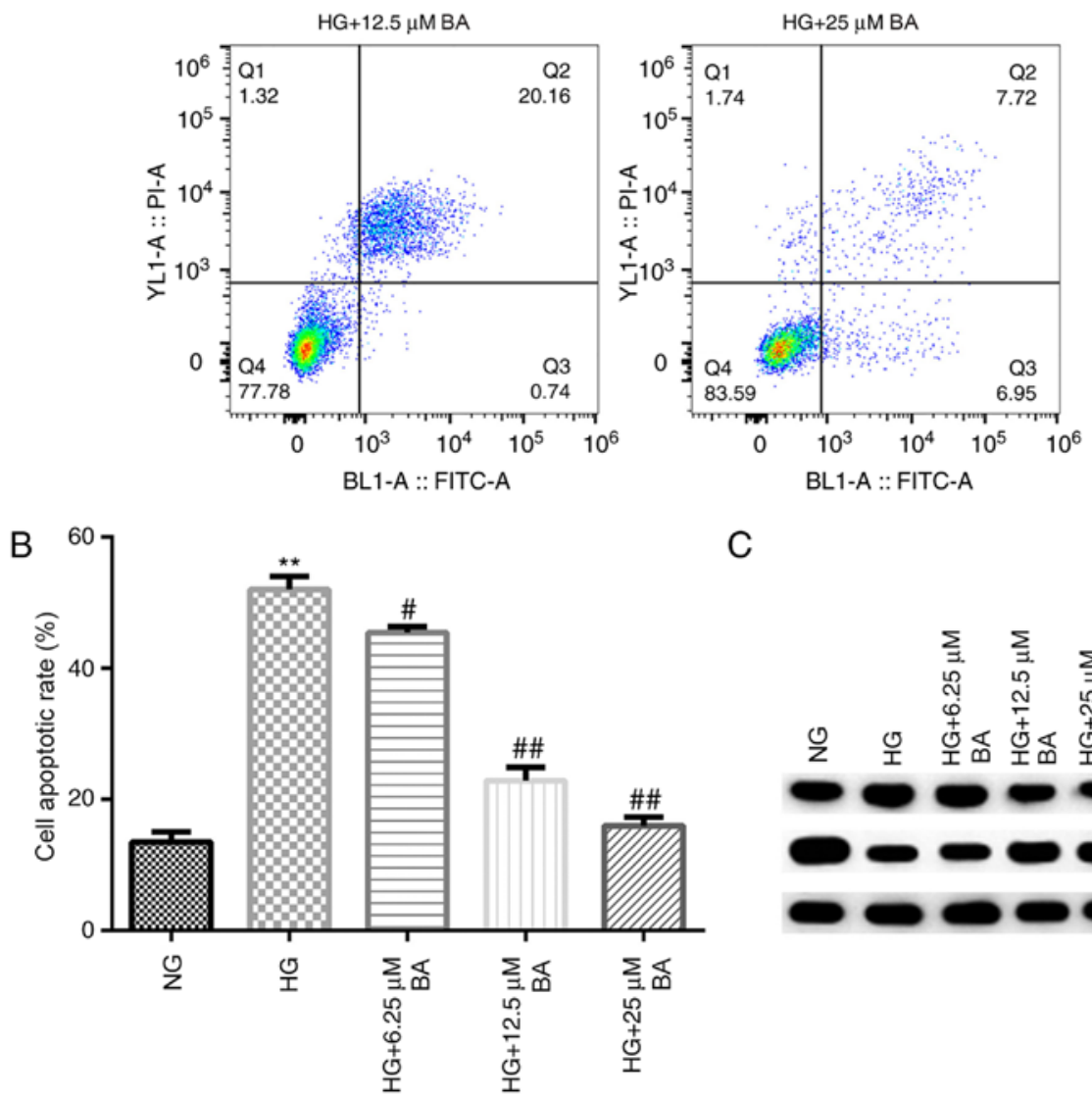

C

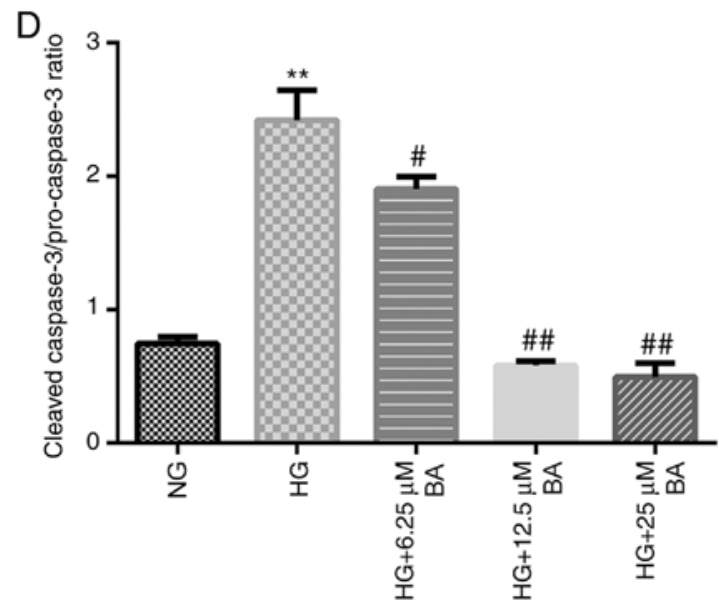

Figure 3. BA improves HG-induced podocyte apoptosis. Following serum starvation for $12 \mathrm{~h}$, podocytes were cultured with HG (30 mM D-glucose) or normal glucose (5 mM D-glucose) for $48 \mathrm{~h}$ and then treated with $6.25,12.5$ or $25 \mu \mathrm{M} \mathrm{BA}$ for $24 \mathrm{~h}$. (A and B) Flow cytometry was used to determine the cell apoptotic rate. (C) Western blotting was performed to determine the protein expression levels of the apoptosis-associated proteins pro-caspase- 3 and cleaved caspase- 3 . (D) Semi-quantification of the ratio between cleaved-caspase-3/pro-caspase-3. All data are presented as the mean $\pm \mathrm{SD}$. ${ }^{* *} \mathrm{P}<0.01$ vs. NG group. ${ }^{\#} \mathrm{P}<0.05$ and ${ }^{\#} \mathrm{P}<0.01$ vs. HG treatment group. BA, baicalin; HG, high glucose; PI, propidium iodide; NG, normal glucose. 

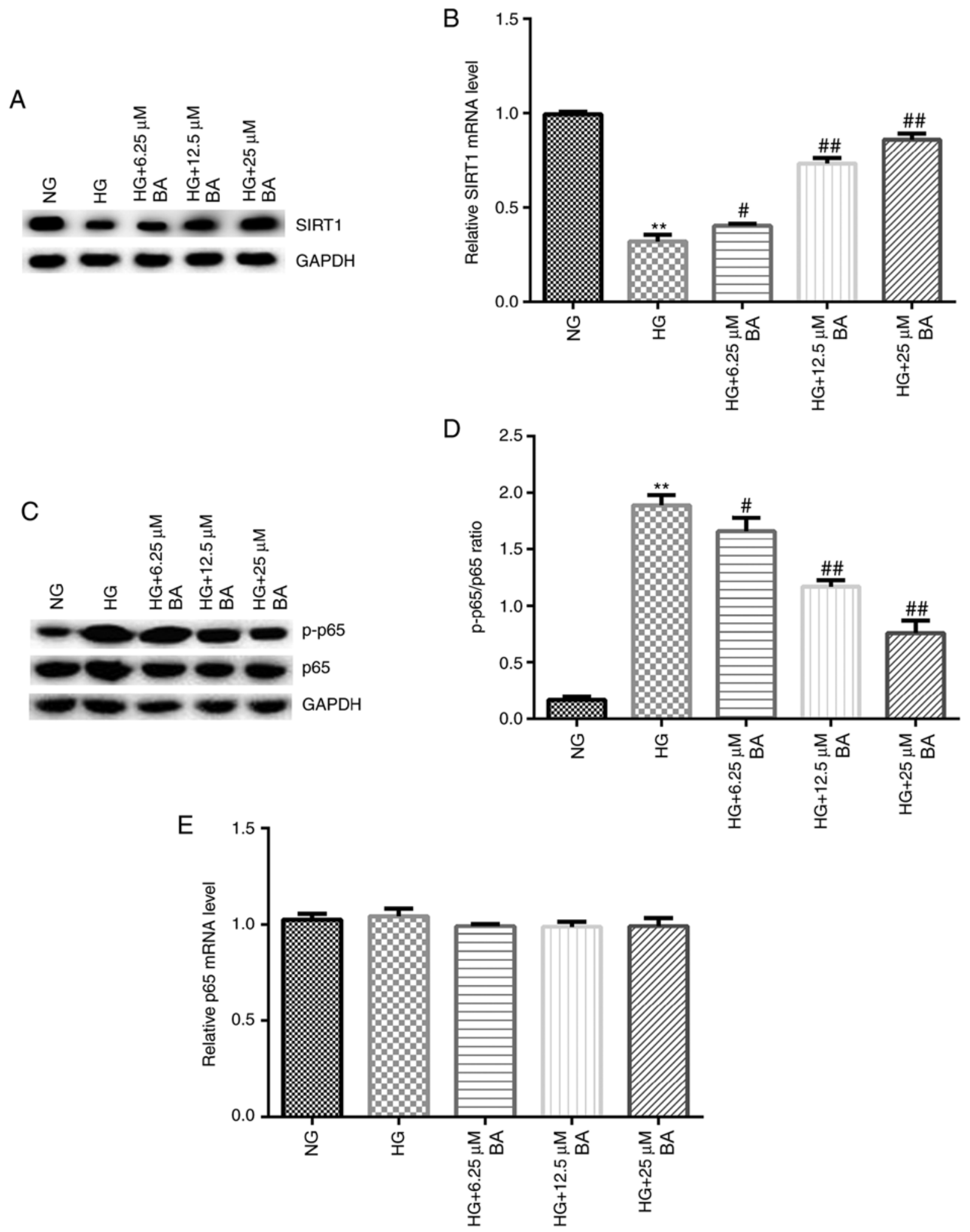

Figure 4. BA increases the expression levels of SIRT1 and p-p65 in HG-induced podocytes. Following serum starvation for $12 \mathrm{~h}$, podocytes were cultured with HG (30 mM D-glucose) or normal glucose (5 mM D-glucose) for $48 \mathrm{~h}$ and then treated with $6.25,12.5$ or $25 \mu \mathrm{M}$ BA for $24 \mathrm{~h}$. (A) Western blotting was used to analyze the expression levels of SIRT1 at the protein level. (B) mRNA expression levels of SIRT1 in podocytes were determined using RT-qPCR. (C) Western blotting was used to analyze the protein expression levels of p-p65 and p65. (D) Semi-quantification of the ratio between p-p65/p65. (E) mRNA expression levels of $\mathrm{p} 65$ in podocytes were analyzed using RT-qPCR. All data are presented as the mean $\pm \mathrm{SD}$. ${ }^{* *} \mathrm{P}<0.01$ vs. NG group. ${ }^{*} \mathrm{P}<0.05$ and ${ }^{\# \prime \prime} \mathrm{P}<0.01$ vs. HG treatment group. BA, baicalin; HG, high glucose; p, phosphorylated; RT-qPCR, reverse transcription-quantitative PCR; SIRT1, sirtuin 1; NG, normal glucose.

the expression levels of apoptosis-associated proteins, respectively. These results indicated that compared with the NG group, HG stimulation significantly increased the apoptotic rate of podocytes (Fig. 3A and B) and enhanced the cleaved-caspase-3/pro-caspase-3 ratio (Fig. 3C and D). Moreover, compared with that in the HG group, BA 
treatment significantly decreased the rate of apoptosis in podocytes in a dose-dependent manner and the reduced cleaved-caspase-3/pro-caspase-3 ratio (Fig. 3C and D).

Effects of BA on the expression levels of SIRT1 and p-p65 in $H G$-induced podocytes. RT-qPCR and western blotting assays were performed to detect the expression of SIRT1 and p-p65 mRNA and protein, respectively. Compared with the NG group, HG-induced podocytes exhibited decreased SIRT1 protein expression levels (Fig. 4A) and significantly decreased SIRT1 mRNA expression levels (Fig. 4B). Moreover, compared with the results in the control group, HG stimulation increased p-p65 protein expression levels (Fig. 4C) and the p-p65/p65 ratio (Fig. 4D). Notably, BA treatment increased the expression levels of SIRT1 protein and mRNA in a dose-dependent manner (Fig. 4A and B), whilst decreasing p-p65 protein expression levels (Fig. 4C) and the ratio between p-p65/p65 compared with the HG group (Fig. 4D). There were no significant differences observed in the expression levels of p65 at the protein or mRNA level between the groups (Fig. 4C and E).

\section{Discussion}

At present, the main risk of diabetes is the occurrence of chronic complications, for example, high levels of blood glucose can lead to damage in the retina, nerves and kidneys (25). Therefore, it is important to prevent the occurrence of such complications. DN is the most important chronic complication of patients with diabetes and it demonstrates a complex pathogenesis, including the involvement of genetic factors and the activation of polyol signaling pathways, the inflammatory response and the oxidative stress response (1).

BA, a natural molecule, is a major bioactive flavonoid (26). Multiple studies have demonstrated that BA serves a variety of biological functions, including antibacterial (27), antiviral (28-30), antitumor (31) and anti-inflammatory effects $(32,33)$. For example, Lu et al (34) identified that BA promoted osteoclast maturation and function through the MAPK/microphthalmia-associated transcription factor signaling pathway; Zheng et al (35) suggested that BA may serve as a potential therapeutic against leukemia; and $\mathrm{Ju}$ et al (36) revealed that BA protected against thrombin-induced cell damage by inhibiting the expression of proteinase-activated receptor 1 and $\mathrm{NF}-\kappa \mathrm{B}$ activation. However, the role of $\mathrm{BA}$ in DN remains relatively unclear. Therefore, the present study investigated the effects of BA on HG-induced podocytes. The results demonstrated that BA significantly improved podocyte viability and decreased the rate of cell apoptosis. Following the establishment of a HG-induced podocyte injury model, it was also revealed that BA significantly increased the viability of HG-induced podocyte cells and decreased the rate of cell apoptosis.

Previous studies have reported that SIRTs were highly expressed and activated in the kidney, liver, spleen, lung, heart and muscle $(37,38)$. In addition, SIRT was identified to be heterogeneous, serving different physiological and pathological roles in different cells and tissues under certain stress conditions (39). SIRT1 is the most studied member of the SIRT family, which is most likely due to its ability to regulate the cell cycle, mitochondrial metabolism, energy homeostasis, inflammation, oxidative stress and apoptosis (38). SIRT1 was found to serve a crucial role in systemic metabolic homeostasis; the downregulation of SIRT1 expression in visceral adipose tissue led to metabolic abnormalities associated with visceral obesity in diabetic and obese women (40). In the present study, BA treatment increased SIRT1 expression levels in a dose-dependent manner in HG-induced podocytes. In addition, BA treatment decreased the p-p65/p65 ratio, whilst there were no significant differences observed in the expression levels of total p65 at both the protein and mRNA level between BA-treated cells and the control cells. Thus, these data indicated that BA may inhibit the HG-induced activation of the NF- $\kappa$ B signaling pathway. Previous studies have demonstrated that the high expression levels of SIRT1 decreased the acetylation of NF- $\kappa \mathrm{B}$ and decreased the side effects of cisplatin on renal tubular toxicity (41). Taken together, it was suggested that BA may suppress HG-induced podocyte apoptosis in DN. However, it is worth noting that in the present study, the apoptotic rate of the NG group was $>10 \%$, which may just represent the normal rate of programmed cell death in podocytes or it may be due to the cell culture environment; thus, further confirmation is required. Another interesting result was that in contrast with our study, a previous study reported that BA induced apoptosis in cancer cells (10-12).

In conclusion, the results of the present study suggested that BA treatment may inhibit podocyte apoptosis in a $\mathrm{HG}$ environment and serve a protective role in DN. In addition, the mechanism of action of BA may be associated with its regulation over SIRT1/NF- $\kappa$ B signaling pathway activation. However, this study is only a preliminary in vitro study of the role of BA in DN. To further validate the role of BA in DN, more in-depth studies are required; for example, determining

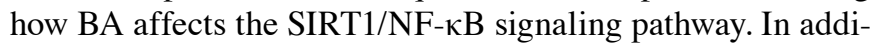
tion, the effect of BA in DN needs to be verified in vivo, which is our aim in future studies.

\section{Acknowledgements}

Not applicable.

\section{Funding}

The present study was partly supported by Research Foundation of Taizhou People's Hospital (grant no. ZL201951).

\section{Availability of data and materials}

The datasets used and/or analyzed during the present study are available from the corresponding author on reasonable request.

\section{Authors' contributions}

JL designed the study, collected the data, performed the statistical analysis, interpreted the data and prepared the manuscript. YL, SYi, SYa and MK contributed to the data collection and helped perform the statistical analysis. ZL contributed to the data collection, statistical analysis and helped prepare the manuscript. All authors read and approved the final manuscript. 


\section{Ethics approval and consent to participate}

Not applicable.

\section{Patient consent for publication}

Not applicable.

\section{Competing interests}

The authors declare that they have no competing interests.

\section{References}

1. Yang M, Kan L, Wu L, Zhu Y and Wang Q: Effect of baicalin on renal function in patients with diabetic nephropathy and its therapeutic mechanism. Exp Ther Med 17: 2071-2076, 2019.

2. Akhtar M, Taha NM,Nauman A,Mujeeb IB and Al-Nabet ADMH: Diabetic kidney disease: Past and present. Adv Anat Pathol 27: 87-97, 2020.

3. Fu J, Lee K, Chuang PY, Liu Z and He JC: Glomerular endothelial cell injury and cross talk in diabetic kidney disease. Am J Physiol Renal Physiol 308: F287-F297, 2017.

4. Nath KA: Tubulointerstitial changes as a major determinant in the progression of renal damage. Am J Kidney Dis 20: 1-17, 1992.

5. Kowalski A, Krikorian A and Lerma EV: Diabetic nephropathy for the primary care provider: New understandings on early detection and treatment. Ochsner J 14: 369-379, 2014.

6. Papadopoulou-Marketou N, Kanaka-Gantenbein C, Marketos N, Chrousos GP and Papassotiriou I: Biomarkers of diabetic nephropathy: A 2017 update. Crit Rev Clin Lab Sci 54: 326-342, 2017.

7. Ying Q and $\mathrm{Wu} \mathrm{G}$ : Molecular mechanisms involved in podocyte EMT and concomitant diabetic kidney diseases: An update. Ren Fail 39: 474-483, 2017.

8. Chen J, Chen JK and Harris RC: EGF receptor deletion in podocytes attenuates diabetic nephropathy. J Am Soc Nephrol 26 $1115-1125,2015$.

9. Luo J, Dong B, Wang K, Cai S, Liu T, Cheng X, Lei D, Chen Y, Li Y, Kong $\mathrm{J}$ and Chen Y: Baicalin inhibits biofilm formation, attenuates the quorum sensing-controlled virulence and enhances Pseudomonas aeruginosa clearance in a mouse peritoneal implant infection model. PLoS One 12: e0176883, 2017.

10. Jia Y, Chen L, Guo S and Li Y: Baicalin induced colon cancer cells apoptosis through miR-217/DKK1-mediated inhibition of wnt signaling pathway. Mol Biol Rep 46: 1693-1700, 2019.

11. Wang XF, Zhou QM, Du J, Zhang H, Lu YY and Su SB: Baicalin suppresses migration, invasion and metastasis of breast cancer via p38MAPK signaling pathway. Anticancer Agents Med Chem 13: 923-931, 2013.

12. Cao HJ, Yan YB, Dai JY, et al: Effect of baicalin on human lung cancer A549 cell line. Chin J Exp Tradit Med Formul 19: 216-220, 2013 (In Chinese)

13. Yi QQ, Xia YY, Chen J, et al: Baicalin improves diabetic nephropathy in mice by activating Sirt1/Nrf2 signal through miR-141 inhibition. Med J Wuhan Uni 40: 186-191, 2019 (In Chinese).

14. Sun QR, Zhang X and Fang K: Phenotype of vascular smooth muscle cells (VSMCs) is regulated by miR-29b by targeting sirtuin 1. Med Sci Monit 24: 6599-6607, 2018.

15. Morigi M, Perico L and Benigni A: Sirtuins in renal health and disease. J Am Soc Nephrol 29: 1799-1809, 2018.

16. Kitada M, Kume S, Takeda-Watanabe A, Kanasaki K and Koya D: Sirtuins and renal diseases: Relationship with aging and diabetic nephropathy. Clin Sci (Lond) 124: 153-164, 2013.

17. Shen $\mathrm{HM}$ and Tergaonkar V: NF- $\kappa \mathrm{B}$ signaling in carcinogenesis and as a potential molecular target for cancer therapy. Apoptosis 14: 348-363, 2009.

18. Deng X, Sun L, Lai X, Xiang L, Li Q, Zhang W, Zhang L and Sun S: Tea polypeptide ameliorates diabetic nephropathy through RAGE and NF- $\kappa$ B signaling pathway in type 2 diabetes mice. J Agric Food Chem 66: 11957-11967, 2018.

19. Liu T, Zhang L, Joo D and Sun SC: NF- $\kappa B$ signaling in inflammation. Signal Transduct Target Ther 2: 17023, 2017.

20. Kuhad A and Chopra K: Attenuation of diabetic nephropathy by tocotrienol: Involvement of NF-kB signaling pathway. Life Sci 84: 296-301, 2009.
21. Zhu L, Han J, Yuan R, Xue L and Pang W: Berberine ameliorates diabetic nephropathy by inhibiting TLR4/NF- $\kappa$ B pathway. Biol Res 51: 9, 2018

22. Xu L, Zhang P, Guan H, Huang Z, He X, Wan X, Xiao H and Li Y: Vitamin $\mathrm{D}$ and its receptor regulate lipopolysaccharide-induced transforming growth factor- $\beta$, angiotensinogen expression and podocytes apoptosis through the nuclear factor $-\kappa B$ pathway. J Diabetes Investig 7: 680-688, 2016.

23. Zhang SZ, Qiu XJ, Dong SS, Zhou LN, Zhu Y, Wang MD and Jin LW: MicroRNA-770-5p is involved in the development of diabetic nephropathy through regulating podocyte apoptosis by targeting TP53 regulated inhibitor of apoptosis 1. Eur Rev Med Pharmacol Sci 23: 1248-1256,2019.

24. Livak KJ and Schmittgen TD: Analysis of relative gene expression data using real-time quantitative PCR and the 2(-Delta Delta C(T)) method. Methods 25: 402-408, 2001.

25. Kawada T: Risk factors for developing prediabetes. Diabetes Res Clin Pract 135: 232, 2018.

26. Peng XD, Dai LL, Huang CQ, He CM and Chen LJ: Correlation between anti-fibrotic effect of baicalin and serum cytokines in rat hepatic fibrosis. World J Gastroenterol 15: 4720-4725, 2009.

27. Roy MK, Nakahara K, Na TV, Trakoontivakorn G, Takenaka M, Isobe $\mathrm{S}$ and Tsushida T: Baicalein, a flavonoid extracted from a methanolic extract of oroxylum indicum inhibits proliferation of a cancer cell line in vitro via induction of apoptosis. Pharmazie 62: 149-153, 2007.

28. Li HY, Hu J, Zhao S, Yuan ZY, Wan HJ, Lei F, Ding Y, Xing DM and Du LJ: Comparative study of the effect of baicalin and its natural analogs on neurons with oxygen and glucose deprivation involving innate immune reaction of TLR2/TNF- $\alpha$. J Biomed Biotechnol 2012: 267890, 2012.

29. Moghaddam E, Teoh BT, Sam SS, Lani R, Hassandarvish P, Chik Z, Yueh A, Abubakar S and Zandi K: Baicalin, a metabolite of baicalein with antiviral activity against dengue virus. Sci Rep 4: 5452, 2014.

30. Nayak MK, Agrawal AS, Bose S, Naskar S, Bhowmick R, Chakrabarti S, Sarkar S and Chawla-Sarkar M: Antiviral activity of baicalin against influenza virus H1N1-pdm09 is due to modulation of NS1-mediated cellular innate immune responses. J Antimicrob Chemoth 69: 1298-1310, 2014.

31. Chen J, Li Z, Chen AY, Ye X, Luo H, Rankin GO and Chen YC: Inhibitory effect of baicalin and baicalein on ovarian cancer cells. Int J Mol Sci 14: 6012-6025, 2013.

32. Novy P, Urban J, Leuner O, Vadlejch J and Kokoska L: In vitro synergistic effects of baicalin with oxytetracycline and tetracycline against Staphylococcus aureus. J Antimicrob Chemoth 66: 1298-1300, 2011.

33. Lee W, Ku SK and Bae JS: Anti-Inflammatory effects of baicalin, baicalein, and wogonin in vitro and in vivo. Inflammation 38: 110-125, 2015.

34. Lu L, Rao L, Jia H, Chen J, Lu X, Yang G, Li Q, Lee KKH and Yang L: Baicalin positively regulates osteoclast function by activating MAPK/Mitf signaling. J Cell Mol Med 21: 1361-1372, 2017.

35. Zheng J, Asakawa T, Chen Y,Zheng Z, Chen B,Lin M,Liu T and Hu J: Synergistic effect of baicalin and adriamycin in resistant HL-60/ADM leukaemia cells. Cell Physiol Biochem 43: 419-430, 2017.

36. Ju XN, Mu WN, Liu YT, Wang MH, Kong F, Sun C and Zhou QB: Baicalin protects against thrombin induced cell injury in SH-SY5Y cells. Int J Clin Exp Pathol 8: 14021-14027, 2015.

37. Morigi M, Perico L and Benigni A: Sirtuins in renal health and disease. J Am Soc Nephrol 29: 1799-1809, 2018.

38. Chang HC and Guarente L: SIRT1 and other sirtuins in metabolism. Trends Endocrinol Metab 25: 138-145, 2014.

39. Dai H, Sinclair DA, Ellis JL and Steegborn C: Sirtuin activators and inhibitors: Promises, achievements, and challenges. Pharmacol Ther 188: 140-154, 2018.

40. Bible E: Diabetic nephropathy: Sirt1 attenuates diabetic albuminuria. Nat Rev Nephrol 9: 696, 2013.

41. Sakao Y, Kato A, Tsuji T, Yasuda H, Togawa A, Fujigaki Y, Kahyo T, Setou M and Hishida A: Cisplatin induces Sirtl in association with histone deacetylation and increased Werner syndrome protein in the kidney. Clin Exp Nephrol 15: 363-372, 2011.

This work is licensed under a Creative Commons Attribution-NonCommercial-NoDerivatives 4.0 International (CC BY-NC-ND 4.0) License. 